\title{
Large-scale structure non-Gaussianities with modal methods
}

\author{
Marcel Schmittfull \\ Berkeley Center for Cosmological Physics, \\ Department of Physics and Lawrence Berkeley National Laboratory, \\ University of California, Berkeley, CA 94720, USA \\ email: mschmittfull@lbl.gov
}

\begin{abstract}
Relying on a separable modal expansion of the bispectrum, the implementation of a fast estimator for the full bispectrum of a $3 \mathrm{~d}$ particle distribution is presented. The computational cost of accurate bispectrum estimation is negligible relative to simulation evolution, so the bispectrum can be used as a standard diagnostic whenever the power spectrum is evaluated. As an application, the time evolution of gravitational and primordial dark matter bispectra was measured in a large suite of N-body simulations. The bispectrum shape changes characteristically when the cosmic web becomes dominated by filaments and halos, therefore providing a quantitative probe of $3 \mathrm{~d}$ structure formation. Our measured bispectra are determined by $\sim 50$ coefficients, which can be used as fitting formulae in the nonlinear regime and for non-Gaussian initial conditions. We also compare the measured bispectra with predictions from the Effective Field Theory of Large Scale Structures (EFTofLSS).
\end{abstract}

Keywords. cosmology: large-scale structure of universe, methods: n-body simulations, methods: data analysis, methods: statistical

Due to non-linear gravitational collapse, the probability distribution function (pdf) of large-scale structure (LSS) dark matter (DM) density fluctuations deviates from a Gaussian pdf even if the initial conditions are Gaussian. This non-Gaussianity is characterized by non-vanishing higher-order $n$-point functions, e.g. the bispectrum, which is the Fourier transform of the 3 -point function and corresponds to the probability of finding a third overdensity mode given two other overdensity modes. An additional source of nonGaussianity is primordial non-Gaussianity of the initial conditions that can be generated by certain inflation models. Measuring these non-Gaussianities in observations can help to break degeneracies present at the level of 2-point statistics, e.g. between linear bias $b_{1}$ and the normalization of fluctuations $\sigma_{8}$, or to constrain inflation models.

While it is numerically straightforward to sample initial conditions from a Gaussian pdf and to estimate the power spectrum of a given density, sampling from a non-Gaussian pdf and estimating the bispectrum of a given density perturbation are numerically rather challenging tasks. We address these issues by expanding the bispectrum in separable basis functions which are particularly suited for efficient numerical evaluation.

\section{Non-Gaussian initial conditions for $N$-body simulations}

To draw a realization from a pdf with power $P_{\Phi}$ and bispectrum $f_{\mathrm{NL}} B_{\Phi}$, we add $\Phi^{B}=\int_{\mathbf{k}^{\prime}} W\left(k, k^{\prime},\left|\mathbf{k}-\mathbf{k}^{\prime}\right|\right) \Phi_{\mathbf{k}^{\prime}}^{G} \Phi_{\mathbf{k}-\mathbf{k}^{\prime}}^{G}$ to a Gaussian field $\Phi_{\mathbf{k}}^{G}$, where (Wagner et al. (2012))

$$
W\left(k, k^{\prime},\left|\mathbf{k}-\mathbf{k}^{\prime}\right|\right) \equiv \frac{f_{\mathrm{NL}}}{2} \frac{B_{\Phi}\left(k, k^{\prime},\left|\mathbf{k}-\mathbf{k}^{\prime}\right|\right)}{P_{\Phi}(k) P_{\Phi}\left(k^{\prime}\right)+2 \text { perms }} .
$$


In practice, $\Phi^{B}$ can only be computed efficiently if $W$ is product-separable, i.e. consisting of terms of the form $f_{1}(k) f_{2}\left(k^{\prime}\right) f_{3}\left(k^{\prime \prime}\right)$, because then $\Phi^{B}$ reduces to a convolution of filtered Gaussian fields. Unfortunately, the symmetrized denominator in (1.1) often destroys separability. As a general solution to this problem, the kernel $W$ can be expanded in product-separable basis functions following Fergusson et al. (2012), which we show in Regan et al. (2012) to perform well in simulations.

\section{Separable bispectrum estimation}

Estimating the bispectrum of a density perturbation is computationally expensive due to the large number of possible triangle configurations and integrals similar to the expression for $\Phi^{B}$ above. Rather than estimating the bispectrum individually for every triangle, Fergusson et al. (2012) propose to estimate the amplitude of many independent separable basis bispectrum templates and reconstruct the full bispectrum by summing up the contributions. We restrict ourselves to a finite separable monomial basis that covers all theoretically motivated bispectra and implicitly uses all triangles. This reduces the computational cost of bispectrum estimation dramatically, e.g. the full bispectrum of a $1024^{3}$ grid is estimated in only one hour on six cores.

We implemented this method and tested it on a large suite of $N$-body simulations with Gaussian and different types of non-Gaussian initial conditions in Schmittfull et al. (2013). All bispectrum measurements agree with perturbation theory on large scales, validating our framework and implementation. In the non-linear regime, we find that the bispectrum shape characterizes the $3 \mathrm{~d}$ dark matter structures, e.g. pancake-like structures correspond to flattened bispectra, while filaments and clusters enhance equilateral contributions to the bispectrum. The bispectrum characterizes these structures in a quantitative way which can both be modeled and extracted from simulations.

In our approach, the full bispectrum information is compressed to $n_{\max }=\mathcal{O}(50)$ amplitudes of (orthonormalized) basis bispectrum shapes. The first ten modes already contain $99 \%$ of the information, which we exploit to construct fitting formulae for the dark matter bispectrum with ten parameters at every redshift and $k_{\max }$. The effect of primordial non-Gaussianity can be modeled by a time shift compared to gravitational evolution in a universe with Gaussian initial conditions, which leads to simple fitting formulae for the excess bispectrum due to primordial non-Gaussianity of various types (see Schmittfull et al. (2013) for details).

In another application of the separable bispectrum estimation method, we compare the measured bispectra against predictions of Effective Field Theory of Large-Scale Structure (EFTofLSS) in Angulo et al. (2014) (see also Baldauf et al. (2014)), finding that EFTofLSS significantly improves upon standard perturbation theory.

Acknowledgements I thank my collaborators for the work summarized here, R. Angulo, J. Fergusson, S. Foreman, D. Regan, L. Senatore and P. Shellard.

\section{References}

Angulo, R., Foreman, S., Schmittfull, M., \& Senatore, L. 2014, arXiv 1406.4143

Baldauf, T., Mercolli, L., Mirbabayi, M., \& Pajer, E. 2014, arXiv 1406.4135

Fergusson, J., Regan, D., \& Shellard, E. P. S.. 2012, Phys. Rev. D 86, 063511, 1008.1730

Regan, D., Schmittfull, M., Shellard, E. P. S., \& Fergusson, J. 2012, Phys. Rev. D 86, 123524, 1108.3813

Schmittfull, M., Regan, D., \& Shellard, E. P. S.. 2013, Phys. Rev. D 88, 063512, 1207.5678

Wagner, C. \& Verde, L. 2012, JCAP 3, 2, 1102.3229 